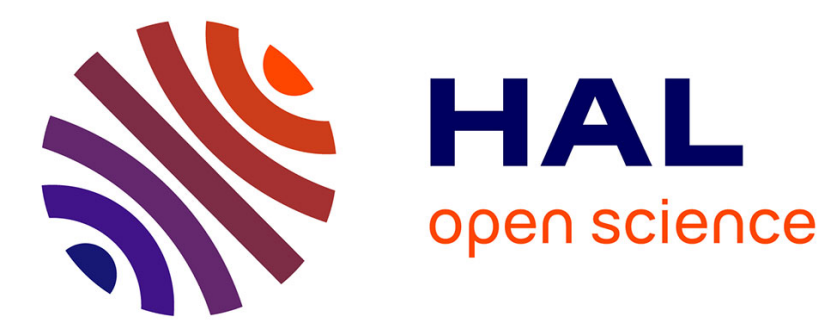

\title{
Techniques nouvelles de filtrage des données H.P.D
}

Ch. de La Vaissière

\section{To cite this version:}

Ch. de La Vaissière. Techniques nouvelles de filtrage des données H.P.D. Revue de Physique Appliquée, 1969, 4 (2), pp.321-322. 10.1051/rphysap:0196900402032100 . jpa-00243282

\section{HAL Id: jpa-00243282 https://hal.science/jpa-00243282}

Submitted on 1 Jan 1969

HAL is a multi-disciplinary open access archive for the deposit and dissemination of scientific research documents, whether they are published or not. The documents may come from teaching and research institutions in France or abroad, or from public or private research centers.
L'archive ouverte pluridisciplinaire HAL, est destinée au dépôt et à la diffusion de documents scientifiques de niveau recherche, publiés ou non, émanant des établissements d'enseignement et de recherche français ou étrangers, des laboratoires publics ou privés. 


\title{
TECHNIQUES NOUVELLES DE FILTRAGE DES DONNÉES H.P.D.
}

\author{
Ch. DE LA VAISSIÈRE, \\ Institut de Physique Nucléaire, Paris.
}

\begin{abstract}
Résumé. - Quelques techniques pour le filtrage " off-line " des données H.P.D., utilisant les corrélations géométriques entre les trois vues d'un même événement, sont proposées. Le filtrage est rendu moins dépendant de la prémesure. Le temps de calcul peut être réduit ou la prémesure.
\end{abstract}

\begin{abstract}
Some techniques using the geometrical correlations in the same frame are proposed for the off-line filtering of H.P.D. data. The filtering is made less dependent on the premeasurements. The computer-time can be reduced, or the guidance of the premeasurements.
\end{abstract}

L'Institut de Physique Nucléaire utilise pour la mesure de clichés de la chambre de $2 \mathrm{~m}$ du G.E.R.N. le système H.P.D. du Collège de France. Les principales caractéristiques du système sont $(f g .1): i)$ une

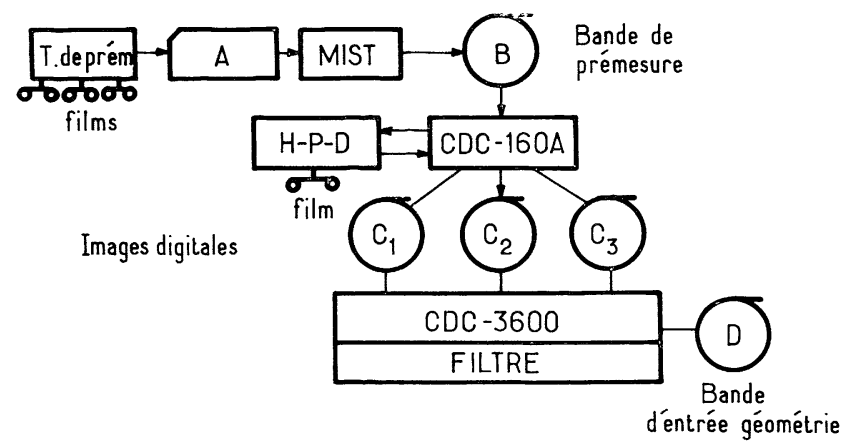

FIG. 1.

Organigramme du système de mesure H.P.D.

mesure scindée en 3 étapes (prémesure, mesure H.P.D., traitement des images digitales); ii) une réduction - environ $75 \%$ du volume de l'image digitale obtenue à la mesure en utilisant les données de prémesure; iii) un filtrage basé sur la notion de route (prémesure d'un apex, et de deux points par trace), effectué « off-line » sur une CDC 3600. Malgré des performances satisfaisantes -3000 événements par semaine mesurés, $70 \%$ des événements acceptés en cinématique - le système présente certains défauts : une prémesure complexe et assez longue : $4 \mathrm{mn}$ par événement; un programme de filtrage trop dépendant de la qualité de la prémesure (une erreur de prémesure $=$ rejet) ; une gestion lourde due au nombre limité d'images digitales pouvant être placé sur bande magnétique $(<250)$; un temps de calcul important : 15 à $20 \mathrm{~s}$ de CDG 3600 par événement.

L'arrivée d'une calculatrice plus puissante REVUE DE PHYSIQUe APPLIQUÉE. - T. 4. No 2. JUIN 1969.
- CDC 6600 - dans un proche avenir à la Faculté des Sciences de Paris, nous a conduit à envisager certaines améliorations qui consistent à abandonner le système des routes interdisant une évolution du filtrage, à réduire la prémesure et à utiliser des constructions géométriques simples pour établir des corrélations entre les traces sur les différentes vues et accélérer le filtrage.

I. Aspects philosophiques d'une réduction de la prémesure. - D'après les résultats préliminaires obtenus, il semble que la donnée d'un point par trace est suffisante pour localiser l'événement sur une vue. Moyennant plus de calcul et de mémoire disponible pour l'image digitale, la prémesure pourrait être réduite à quelques points par événement. Cependant, la réduction des données utilisées actuellement pour diminuer le volume de l'information interdit de descendre en dessous d'un point par trace sur deux vues (au-delà de ce niveau, il n'est plus possible de créer un « masque » valable). L'efficacité du filtrage peut être accrue si l'on utilise les corrélations géométriques entre les projections des traces. La solution « off-line» permet en théorie d'avoir recours aux trois vues. Pour des raisons de place en mémoire et de rapidité, le recours aux trois images digitales n'est possible que si l'on utilise des portions réduites des images et si l'on résume l'information d'une ou de deux images digitales. C'est le cas des traces déjà localisées sur une ou deux vues. Ces considérations nous conduisent à traiter d'une manière différente la première vue des suivantes et à placer en cas d'échec une portion de l'image digitale sur une mémoire externe d'accès rapide de façon à attendre l'information des autres vues pour tenter un nouvel essai. La prémesure est surtout importante pour la vue 1 .

II. Quelques techniques et idées générales. 1) Nous travaillons directement à partir de l'image 21 
digitale. Pour gagner du temps, les digitisations reconnues comme appartenant à une trace sont affectées d'un label. L'examen du label permet de les éliminer d'emblée pour les autres traces. 2) Nous utilisons le caractère circulaire des traces pour localiser un segment ou un élément de trace. 3) Les traces du faisceau sont cherchées à part. L'élimination des traces parallèles dans la région de l'apex permet de soustraire un bruit de fond gênant. 4) Sur la première vue, les traces sont cherchées à partir d'un point approché fourni par la prémesure. On localise d'abord un segment du voisinage de ce point à peu près dirigé vers l'apex. Le cercle tangent à l'élément et passant par l'apex est utilisé pour retrouver la trace en son milieu. Ce point milieu obtenu, la trace est facile à localiser dans son ensemble. 5) Les traces qui s'arrêtent feront l'objet d'un traitement spécial et la longueur des traces mesurées sera déterminée par le programme et non par la prémesure. 6) Une recherche à partir de l'apex est envisagée en cas d'échec d'une trace. Elle est d'autant plus efficace que les autres traces ont été trouvées et marquées.

III. Utilisations de reconstructions géométriques simples. - Après avoir fait coïncider les marques fiducielles appartenant à un même plan, les 3 projections d'un point de la chambre à bulles sur les 3 vues forment un triangle dont la forme ne dépend que de la disposition des caméras. La longueur des côtés est proportionnelle à la distance du point au plan des marques fiducielles, $z$. Cette méthode peut fournir des données approchées sur les traces : 1) La position d'une trace sur une vue, la position de l'apex et d'un point approché sur une autre vue sont connues. Si l'on admet une inclinaison constante de la trace dans l'espace, la direction de la trace au point approché peut être calculée. 2) Un élément de trace a été localisé sur la vue 2. Le calcul de l'inclinaison permet de prévoir le reste de la trace. 3) Une trace est trouvée sur deux vues (fig. 2). La méthode fournit une suite de

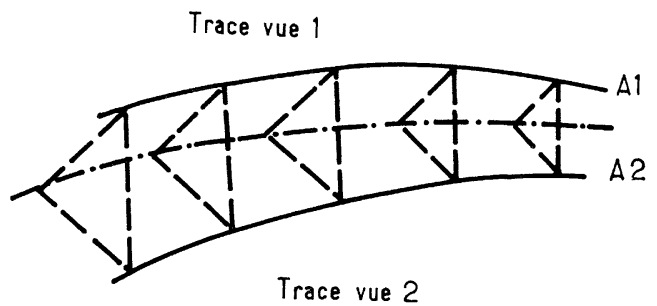

FIG. 2. - Exemple d'application de la méthode des points correspondants. Une trace est localisée sur deux vues. La méthode fournit une suite de points approchés précis.

points correspondants proches de la trace réelle. Le calcul est très rapide. 4) En cas d'échec sur les 3 vues, on peut avoir recours exceptionnellement à 3 portions réduites des images digitales.

IV. Résultats. - Les résultats obtenus montrent que la donnée d'un point approché et d'un apex est suffisante pour localiser 90 à $95 \%$ des traces sur la première vue. Les méthodes de reconstruction géométrique n'ont pas encore été introduites. Si elles se révèlent efficaces, le taux global de rejet pourrait être rendu inférieur à $10 \%$. Un compromis intéressant peut être réalisé entre une réduction de la prémesure et une diminution du temps de calcul. 